\title{
IN2CLOUD: A novel concept for collaborative management of big railway data
}

(C) The Author(s) 2017. Published by Higher Education Press. This is an open access article under the CC BY license (http:// creativecommons.org/licenses/by/4.0)

\begin{abstract}
In the EU Horizon 2020 Shift2Rail MultiAnnual Action Plan, the challenge of railway maintenance is generating knowledge from data and/or information. Therefore, we promote a novel concept called "IN2CLOUD," which comprises three sub-concepts, to address this challenge: 1) A hybrid cloud, 2) an intelligent cloud with hybrid cloud learning, and 3) collaborative management using asset-related data acquired from the intelligent hybrid cloud. The concept is developed under the assumption that organizations want/need to learn from each other (including domain knowledge and experience) but do not want to share their raw data or information. IN2CLOUD will help the movement of railway industry systems from "local" to "global" optimization in a collaborative way. The development of cutting-edge intelligent hybrid cloud-based solutions, including information technology (IT) solutions and related methodologies, will enhance business security, economic sustainability, and decision support in the field of intelligent asset management of railway assets.
\end{abstract}

Keywords railway, intelligent asset management, collaborative learning, big data, hybrid cloud, Bayesian

\section{Introduction: Background and motivation}

Maintenance approach has significantly changed over the last century, with a shift in emphasis from technological to

Received June 6, 2017; accepted September 2, 2017

Jing LIN (ه), Uday KUMAR

Division of Operation and Maintenance Engineering, Luleå University of Technology, Luleå 97187, Sweden; Luleå Railway Research Centre (JVTC), Luleå 97187, Sweden

E-mail: janet.lin@1tu.se techno-economic considerations. Today, maintenance strategies are applied in an integrated way depending on the criticality of assets and cost-effectiveness (Ben-Daya et al., 2015). These strategies include but are not limited to the following: Corrective maintenance, preventive maintenance, predictive maintenance, proactive and passive maintenance, self-maintenance, smart maintenance, collaborative maintenance, and e-Maintenance. The development of these strategies is ongoing, and new requirements are continually created with the increasing complexity of assets and operating conditions.

In Industry $4.0^{1)}$, improved automation technology, including self-awareness and self-learning, is a must (Lee et al., 2014). Assets can already "talk" to each other or "learn" by themselves using the Internet of Things (IoT) frameworks, cyber-physical systems (CPSs), machine to machine communications, and industrial Internet, that is, the integration of complex physical machinery with networked sensors and software in a collaborative community. However, the knowledge of intelligent data analysis and the ability to create asset self-awareness and self-learning remain limited (Lee et al., 2014) and many data are underutilized. These obstacles to the collaborative efficiency of assets limit moving closer to the ultimate goal of intelligent maintenance.

The extensive use of information and communication technologies (ICT) has led to the advent of big data, as well as big reliability data (Meeker and Hong, 2014) and big maintenance data (Zhang et al., 2015; Zhang et al., 2017) in the field of maintenance. In the railway industry, unprecedented rates and scales of data are being generated by a wide array of sources, including sensor-intensive condition monitoring systems, enterprise asset management systems, enterprise resource planning systems, computerized maintenance management systems, and supervisory control and data acquisition systems (Karim et al., 2016; Thaduri et al., 2015). These sources represent

1) The fourth industrial revolution, as defined by the German government in 2011. 
a rapidly expanding means for operation and maintenance research, especially because researchers and practitioners have begun to realize the potential of exploiting hidden value from these data (Galar et al., 2017). This research expansion has also led to the development of the dynamic railway information management system (DRIMS) concept as specified in the EU Horizon 2020 Shift2Rail MultiAnnual Action Plan (S2R MAAP), as shown in Fig. $1^{1)}$. However, as noted in the "S2R MAAP 2016-2017," certain difficulties in current railway maintenance are affecting the knowledge generation from data/information. These difficulties can be characterized as follows.

- The majority of monitoring and measuring systems are designed as independent tools, thereby making it difficult to fuse information and integrate it into the maintenance process.

- Many individual information systems in the EU railway deal with individual and isolated areas of maintenance process, thus hindering the potential of big data analytics.

- Research and innovation results show that maintenance performance is linked to many heterogeneous parameters, but most are not considered in the maintenance process.

- The typically applied maintenance is still periodic preventive maintenance based on previously established good practices, which is simply integrated into targeted interventions with the appearance of faults.

- The stakeholder environment is becoming more complex due to an increasing number of parties with frequent conflicting priorities involved in operation and maintenance.

The S2R MAAP 2016-2017 emphasizes that a challenge for DRIMS data is to "generate knowledge from data and/or information, which is driven by the available domain knowledge valid for life cycle management and intelligent asset maintenance planning, including automatic detection of anomalies, discovery and description of maintenance workflow processes, and implementation of predictive models of decaying infrastructural assets."

Given the industry requirements and improvements in technology, big data management of railway assets must search for "cloud-based business solutions" (Karim et al., 2016). Cloud-based big DRIMS data asset management is a growing trend in economics and data safety. With respect to maintenance, most studies and applications of cloudbased business solutions have been dedicated to manufacturing systems (Yang et al., 2015; Lee et al., 2013). In the railway sector, related applications are mainly for traffic control, such as "IItis as a Service" for GGB in Switzerland ${ }^{2}$. However, this is changing. In Europe, the project "INFRALERT $T^{3) "}$ under the framework of Horizon

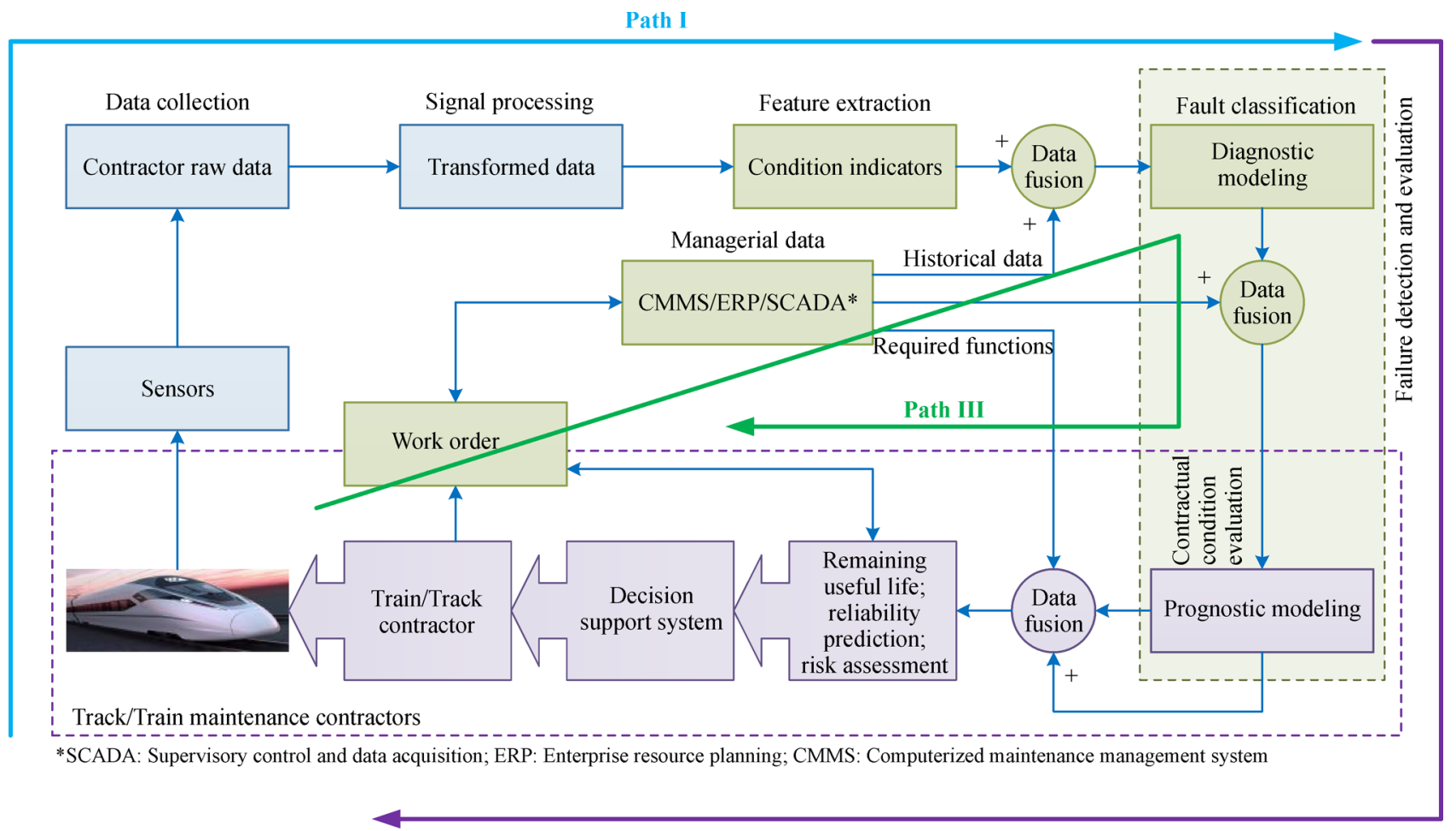

Path II

Fig. 1 Dynamic railway information management system (DRIMS) concept

1) This figure is redrawn by the authors following the EU Horizon 2020 S2R MAAP. The three paths will be discussed later in this paper.

2) https://www.siemens.com/innovation/en/home/pictures-of-the-future/mobility-and-motors/urban-mobility-gornergratbahn.html.

3) http://infralert.eu/ 
2020 is developing a cloud-based information system to support and automate linear asset infrastructure management from measurement to maintenance. In Sweden, the project "ePilot")," which is supported by Luleå Railway Research Center, is conducting research on cloud-based solutions to support railway maintenance for the Swedish Transportation Administration. However, two problems exist. First, the existing cloud-based business solutions are inadequate (Creamer, 2017) and cannot handle a few issues in railway applications; for example, their knowledgegenerating capability is insufficient. Second, although many studies have worked on heterogeneous data formats and complex operation conditions, nearly all organizations want/need to learn from each other, including domain knowledge and experience. In addition, although they all want/need "global optimization" and not only "local optimization," they do not want to share their raw data, information, or even knowledge with each other.

The IN2CLOUD concept has the potential to address both problems. This concept aims to go beyond the current state-of-the-art research by directly addressing the challenges involved in generating knowledge from railway asset maintenance data (e.g., DRIMS data) by developing novel intelligent hybrid cloud-based solutions to facilitate big DRIMS data-driven asset management. These cuttingedge and intelligent hybrid cloud-based solutions, including IT solutions and related methodologies, will enhance business security, economic sustainability, and decision support in the field of big DRIMS data management of railway assets.

The remainder of the paper is organized as follows. Section 2 defines IN2CLOUD and its three sub-concepts. Section 3 fully develops IN2CLOUD using the example of rolling stock wheels. Section 4 elaborates on the philosophy behind IN2CLOUD. Section 5 offers conclusions and comments.

\section{IN2CLOUD}

IN2CLOUD is an acronym for "collaborative management of big railway data asset from the intelligent cloud." Three sub-concepts of big data asset management underpin the IN2CLOUD concept: 1) A hybrid cloud, 2) an intelligent cloud with hybrid cloud learning, and 3) collaborative management using asset-related data from the intelligent hybrid cloud.

This section studies Sweden, Spain, the Netherlands, and the UK because researchers from the four EU countries were involved in IN2CLOUD discussions at the beginning of 2017.

\subsection{Hybrid cloud for big railway data asset management}

In practice, one challenge of knowledge generation from railway maintenance data is that different organizations adopt different tools and methods. On the one hand, given the limitations of time and resources, ensuring consistency in these organizations in a short period is unrealistic. On the other hand, nearly all organizations want to learn more from each other. Although they do not want to share their raw data or information, they still want/need to learn from each other, including domain knowledge and experience.

IN2CLOUD proposes a hybrid cloud structure comprising private clouds and a community cloud to solve these problems. A private cloud is solely operated by a single organization (one country, one company, or one department), while a community cloud shares infrastructure data/ information among several organizations with common concerns with their permission. As shown in Fig. 2, a single organization (or several organizations from one country, several department/branches from one company) may have a private cloud. In each private cloud, DRIMS data assets are managed according to their individual situations (tools, methods, work flows, and rules). In the community cloud, which comprises several private clouds, certain knowledge, information, and/or data are accumulated and shared among community members. Permissions should be defined at the outset, community members should communicate through "bridges," and the security of the hybrid cloud structure should be protected by "walls."

\subsection{Intelligent cloud for hybrid cloud learning}

The proposed hybrid cloud structure will be intelligent because private and community clouds will embrace learning capability, thereby taking us closer to intelligent maintenance. This intelligent hybrid will also embrace cognitive capabilities facilitated by IoT and CPS. Note that this paper introduces the concept of IN2CLOUD; therefore, the CPS structure or other related ICT will not be discussed in detail.

The intelligent hybrid cloud concept provides three basic assumptions. First, partners of the community cloud (who own private clouds) will not be willing to share their knowledge or data/information. Second, community members will be motivated to share some of their knowledge or data/information with permission if the value of their data assets will be increased in the community cloud. Third, the community cloud will perform a different function than private clouds, with private clouds acting as virtual sensors ${ }^{2}$ for the community cloud. As indicated in Fig. 3, inputs from these virtual sensors are different from those embraced by the private

1) https://www.ltu.se/research/subjects/Drift-och-underhall/Forskningsprojekt/ePilot?1 = en.

2) See "Virtual sensing" at https://en.wikipedia.org/wiki/Virtual_sensing. Herein, it means private clouds can act as virtual sensing of community clouds. 


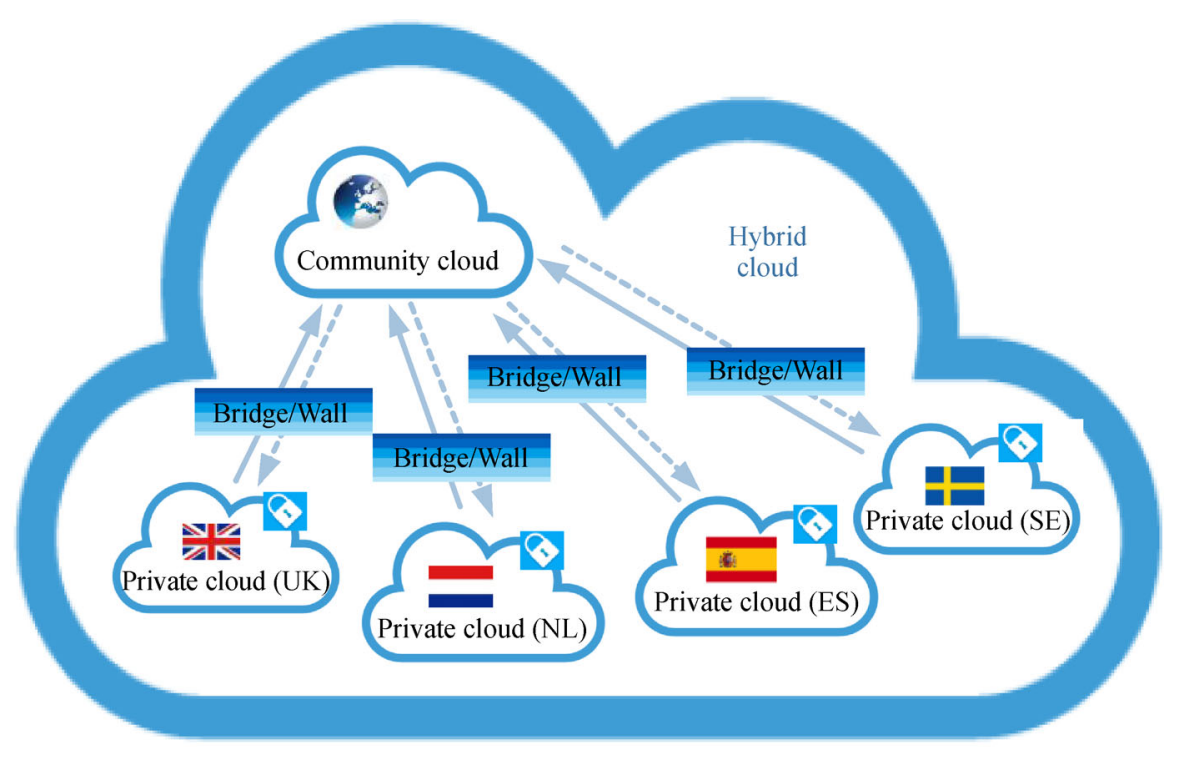

Fig. 2 Hybrid cloud in IN2CLOUD

cloud through real sensors (or big DRIMS data). For example, a few inputs may be obtained from the feedback of various work orders, such as corrective maintenance (CM) work orders, preventive maintenance (PM) work orders, or predictive maintenance (PdM) work orders, instead of original work orders (which are normally stored in private cloud).

The hybrid cloud in Fig. 3 shows that the learning capabilities of various clouds are reflected in increased knowledge, improved models, and good benchmarking. In each private cloud, big DRIMS data will be locally managed according to the situation of that cloud (tools, methods, work flows, and rules). As time passes and historical data grow, local knowledge will accumulate, local models and model parameters will improve, and benchmarking can be implemented using historical records or manufacturing information in these private clouds. Therefore, maintenance strategies can be locally optimized.

However, knowledge, models, and benchmarking can only be further optimized if they can obtain more valuable knowledge or information/data on a large scale, such as from the community cloud in IN2CLOUD. For example, if knowledge from different private clouds can be shared (e.g., stakeholders from Sweden can share knowledge with stakeholders from Spain, the Netherlands, and the UK), then the decision capability of each cloud will be increased, thereby motivating them to participate in data asset "activities" in the community cloud. Models selected from each private cloud can be accumulated and the results can be compared and fused in the community cloud to deal with common problems across clouds. Even if the models in private and community cloud are the same, the historical data/information (e.g., prior information) and parameters of the models will be different; thus, the results will also be different.

In other words, the results in the community cloud will be more robust than the private cloud, thereby permitting global optimization. During this global optimization process, knowledge, information, and data will be generated, stored, and shared through the community clouds. If the community members are willing to perform global benchmarking (e.g., operators of rolling stock wheels in Sweden may want to learn how similar wheels are managed in similar environments in other countries; relevant information could be maintenance strategies, operation reliability, and life cycle cost), certain information and data could be stored and shared through community clouds. Apart from sharing with private clouds, the community cloud itself will learn and grow with time. The parameters of the models in the community cloud will become optimized; in turn, these optimized parameters will become inputs to improve private clouds.

Owing to uncertainties in reality, even in private clouds, no single model can be fitted for all situations. Therefore, in the community cloud, IN2CLOUD will employ machine-learning approaches and categorize neighbors under different rules; this basic idea is similar to anomaly detection in big railway maintenance data analytics (Zhang et al., 2015; Zhang et al., 2017). Bayesian learning ${ }^{1)}$

1) Bayesian learning presents modern methods and techniques for analyzing input information from a Bayesian perspective. Bayesian learning has been attractive to engineers at least half a century ago due to its capability of considering information from past experiments, impressions, and prejudices; in particular, it is time-consuming (or cost-consuming) to obtain sufficient data for output prediction. 


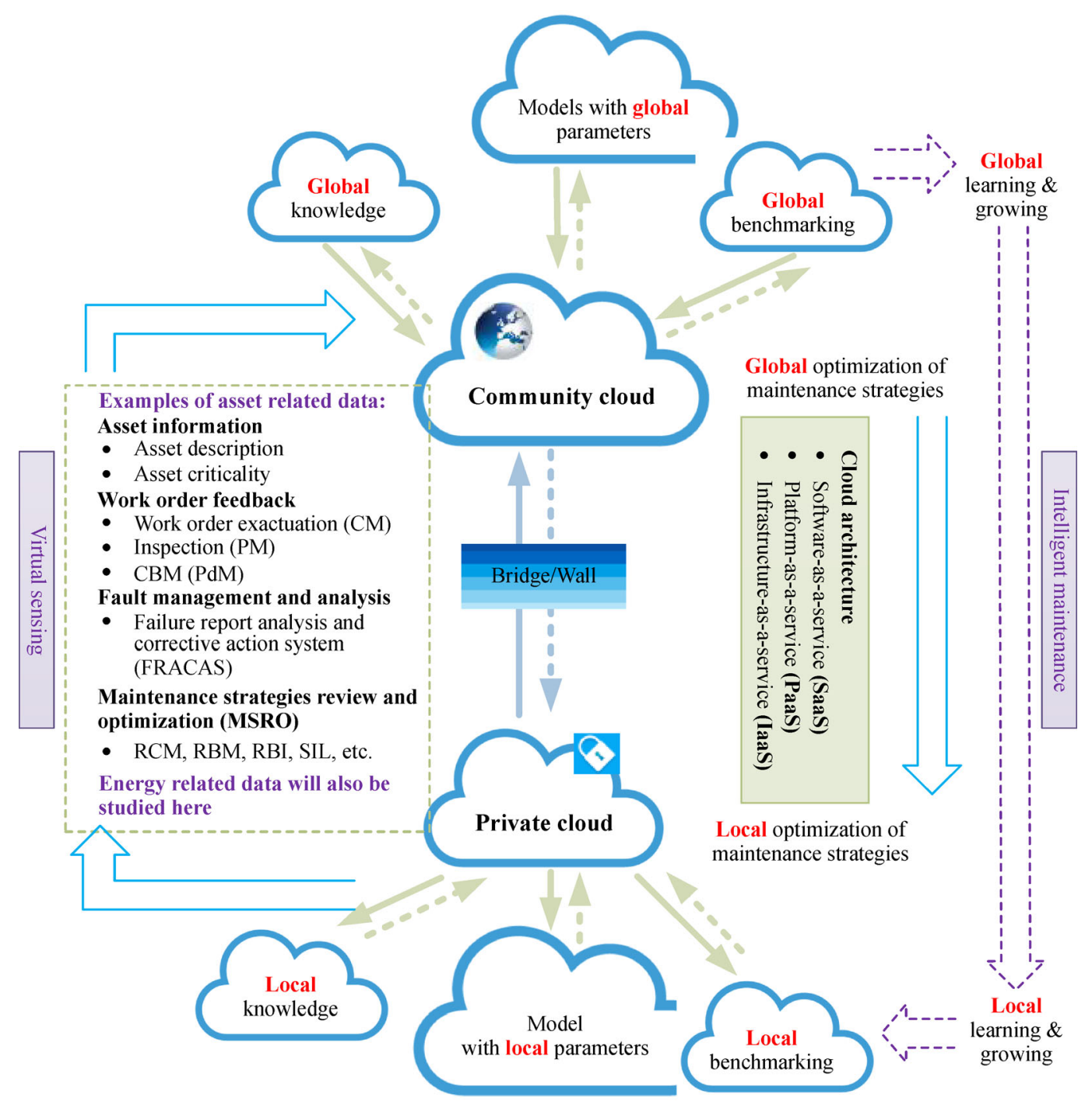

Fig. 3 Intelligent hybrid cloud in IN2CLOUD

approaches would be useful for updating model parameters, models, knowledge, and benchmarking to further achieve global optimizations (a simple example will be discussed later). However, compared with studies that would effectively achieve local optimizations (Cai et al., 2013; Lin et al., 2015; Lin et al., 2014), these approaches should be further studied in a global scope, which will be contributed by this hybrid cloud. Furthermore, the basic philosophy of IN2CLOUD comes from Bayesian learning, wherein all related data/information or knowledge from others become "prior" information.

As the virtual sensors of community clouds, private clouds could provide structural and non-structural data. IN2CLOUD could accommodate structural data comprising asset information, structural work order feedback, and fault management and analysis, as well as results that belong to natural language processing, such as nonstructural work order descriptions (Figueres-Esteban et al.,
2016). Notably, in such virtual sensors, the data may be totally different from the data gathered by real physical sensors in the railway system.

The cloud architecture of IN2CLOUD has three levels:

- Application-as-a-service (AaaS);

- Platform-as-a-service (PaaS);

- Infrastructure-as-a-service (IaaS).

More details of cloud architecture will not be provided in this paper due to time and space constraints. Notably, a few new concepts on this architecture, which have been recently promoted (including maintenance-as-a-service, mobility-as-a-service), should be further studied.

\subsection{Collaborative management concept for railway maintenance}

The term collaborative management was originally used to 
describe management techniques that promote a sense of unity and teamwork among managers and supervisors within an organization. The idea of this management style was to allow managers to combine their strengths with the strengths of other team members, thereby possibly collectively offsetting individual weaknesses.

With the rapid development of ICT, collaborative maintenance techniques began to appear in distributed and interactive-based maintenance approaches. By providing a support framework to ensure that updates are reviewed and appropriate actions are taken, this evolved collaborative maintenance allows system users to collaboratively manage case knowledge updating. However, this relatively new concept requires further study. For one thing, evaluating the collaborative maintenance process may be difficult because it relies on the availability of a large-scale system with an active community of users.

In IN2CLOUD, the collaborative management of railway maintenance will differ from "collaborative maintenance in manufacturing" or "collaborative decisionmaking (CDM)," which is currently popular in air transport. In the IN2CLOUD concept, "collaborations" refer to collaborations among experts, as well as collaborations among heterogeneous pieces of data/ information possibly from different assets; decisionmakers may also come from different sectors and have different purposes. For example, the users of the knowledge/information/data can be railway system designers/constructors, train designers/manufacturers, train operators, customers, maintenance engineers, maintenance contractors, spare parts manufacturers, researchers/consultant/experts, and other stakeholders during the entire life cycle. In this concept, collaborative management is achieved through collaborative learning.

As discussed in the previous section, a collaborative community comprises private clouds and a community cloud in IN2CLOUD. From the perspective of private clouds, collaborative management is mainly reflected in improving knowledge, models (including model and parameters) derived from knowledge, models, and benchmarking results obtained from the community cloud. From the perspective of the community cloud, collaborative management reflects learning and growing, with each private cloud acting as a virtual sensor. Therefore, with IN2CLOUD, intelligent maintenance will require the cooperation and collaboration of different partners, stakeholders, and data/information/knowledge.

Collaborative management could take three paths in IN2CLOUD focusing on big DRIMS data:

- Path I: Collaborative diagnostics;

- Path II: Collaborative prognostics and health manage- ment;

- Path III: Collaborative learning from work orders.

Details of the three paths can be found in Fig. 2.

\section{An example of rolling stock wheels through Path III of DRIMS data}

This section uses an example of rolling stock wheels to explain IN2CLOUD. Note that IN2CLOUD is still under development, and this simplified example is merely used to illustrate the concept.

Figure 4 shows a possible way of collaborative learning from re-profiling wheels using IN2CLOUD and focusing on Path III: Collaborative learning from work orders.

In Sweden, a common PM strategy for railway companies is re-profiling wheel-sets after they have run a certain distance. Re-profiling affects the diameter of a wheel-set; once the diameter is reduced to a pre-specified length, the wheel-set is replaced by a new one. Degradation data from work orders are analyzed to describe degradation behavior and predict reliability and residual life. Information from failure reports on CM needs to be included as well. A maintenance threshold based on safety thresholds and maintenance capability is also part of the PM strategy for re-profiling (Lin et al., 2014; Lin et al., 2015; Lin and Asplund, 2015); such thresholds should be considered in diagnostic modeling because wheel profile data collected from condition monitoring sensors, such as wayside performance measurement systems, stem from condition-based maintenance (CBM) work orders (Asplund and Lin, 2016).

Local maintenance strategies will be optimized over time by using the Bayesian learning approach (Lin et al., 2014; Lin et al., 2015; Lin and Asplund, 2015); for example, prior information can be updated and knowledge and models can be improved. Defined asset information (e.g., asset information, operation information, and other maintenance information) and maintenance information from the private cloud (e.g., faulty analysis, degradation analysis, diagnostics, and prognostics) will be shared in the community cloud. Thus, by communicating with the community cloud (e.g., analyzing degradation of wheels), prior information in the private cloud will become part of a large data set in the community cloud, which goes beyond a local scope ${ }^{1)}$.

Benchmarking in the community cloud will supply more insights. As shown in our past studies in Fig. 5, nearly 90\% of the re-profiling ratio and natural wear rate $^{2)}$ for a few specified heavy haul locomotive wheels in Sweden (private

1) Advantage of Bayesian learning is utilization of "prior" information, which will improve the "posterior" results. The posterior (output) will be robust and reliable by combining prior (input) and current data set (input). However, the posterior (output) depends on elicitation quality of prior knowledge (one input), which encounters new challenge in its acquisition, inspection, fusion, and selection considering big data situations.

2) In our studies, Re-profiling amount = Diameters (before) - Diameters (after); Natural wear = Previous diameters (after) - Current diameters (before); Total wear $=$ Re-profiling amount + Natural wear (Lin et al., 2014; Lin et al., 2015; Lin and Asplund, 2015). 


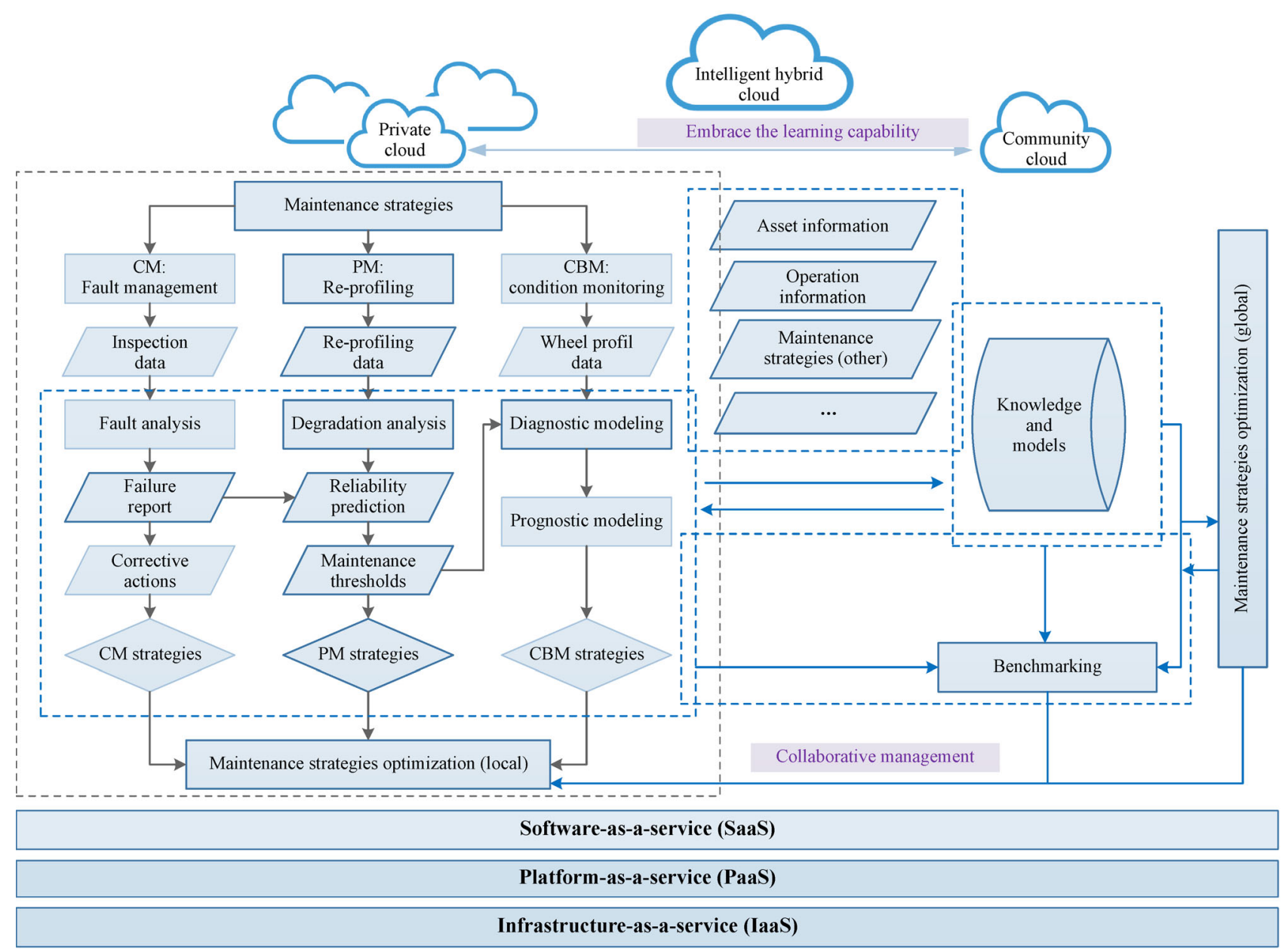

Fig. 4 A possible way of collaborative learning from the re-profiling of wheels

cloud) was two, but knowledge from one private cloud suggests it should be one (in IN2CLOUD, such knowledge will be shared in the community cloud). Therefore, the maintenance threshold can be improved through a Bayesian learning process. In Fig. 5, the original threshold (purple line) will be updated with shared information (original line) in the community cloud. From the perspective of the private cloud, maintenance strategies are collaboratively created with knowledge from the community cloud. From the perspective of the community cloud, results collected from private clouds will collaboratively optimize the knowledge, models, and benchmarking globally.

The final maintenance strategies will be locally optimized because the threshold will be used to integrate CBM data to optimize CBM strategies. As these optimized maintenance strategies perform benchmarking in the community cloud, data/information will be updated globally; therefore, such strategies will also be optimized globally. Other partners will achieve local optimization when they apply these optimized thresholds/maintenance strategies. Taken together, this process leads to continuous improvement via the hybrid cloud. These optimizations will also require new studies on performance evaluation. Researchers have developed a novel performance evaluation approach using the dynamic Bayesian network (Cai et al., 2013), the scope can be expanded globally.

In Sweden, the railway and rolling stock operators belong to different organizations: Swedish Railway Administration and rolling stock owners. Therefore, results from this example, such as the updated degradation behavior of wheel-sets and maintenance threshold, can also be useful for these operators to perform cooperative maintenance by considering various operation situations. For example, as the WPMS (owned by the railway operator) detects the ratios of a few wheel-sets that are larger than one, the information of these wheel-sets should be sent to the train owners to trigger their maintenance thresholds. The ratio should also be updated because the operation situations will be changed, including track situation, axle loads, and speed, in private and community clouds. 


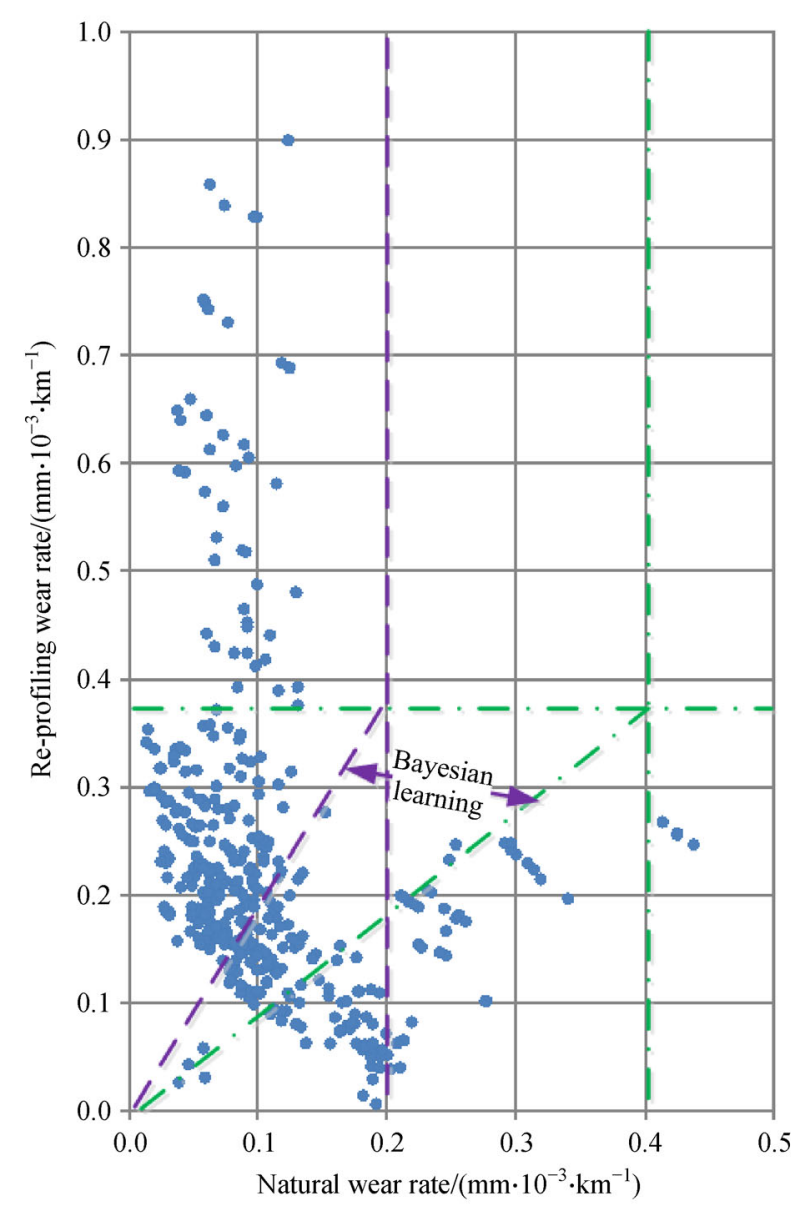

Fig. 5 Updating the threshold in private cloud with shared information in community cloud

\section{Discussion}

The IN2CLOUD concept of collaborative management can change the way of thinking in the railway industry. Collaboration will be accomplished by accommodating various types of data/information, which may be obtained from different assets (similar or different systems), times (historical data or current data), operation situations (different environments, climates, behavior, routes), measurement approaches (different sensors and tools), models (learning models or decision models), and domain knowledge (expert knowledge or engineer experience). Such collaboration will lead to cost-effective and reliable infrastructure.

The basic motivation of IN2CLOUD is the assumption that organizations want/need to learn from each other, including domain knowledge and experience, but do not want to share their raw data or information. However, through IN2CLOUD, the data value of these organizations will be increased, thereby motivating them to share.

The three key aspects of IN2CLOUD are as follows:
Knowledge, models, and benchmarking. Each aspect will have different contents in private and community clouds. In the example provided in Section 3, instead of the reprofiling data on individual wheel-sets stored in private clouds, the community cloud will store a higher level of data/information, such as degradation distributions and maintenance/safety thresholds.

The basic philosophy of IN2CLOUD comes from Bayesian learning, wherein all related data/information or knowledge from others become "prior" information.

IN2CLOUD will increase the capability of knowledgegenerating from data and/or information. Through IN2CLOUD, the learning capabilities of the various clouds are reflected in increased knowledge, improved models, and better benchmarking. Moreover, maintenance strategies can be optimized not only locally but also globally, and results in private and community clouds will be robust. This novel concept will help the movement of railway industry systems from "local" to "global" optimization in a collaborative way.

As a complementary analysis, the metrics and methods/ tools to measure the accuracy of analytic algorithms in all models, including model selection/evaluation, model comparison, and model fusion, should be studied in IN2CLOUD.

\section{Conclusions}

IN2CLOUD focuses on railway infrastructure challenges affecting many European networks and stakeholders. Introducing a collaborative approach and using the now common cloud-based platforms can be groundbreaking. Although the concept is still developing, we believe IN2CLOUD will lead to the creation of new technical solutions and platforms for generating knowledge from big data. More specifically, this concept will address the dynamic improvement of railway asset management by transforming big data collected in various railway systems from asset-related data into knowledge.

The IN2CLOUD concept has the potential to not only increase productivity and assure the economic viability of the entire railway, but it can also be applied to any other organization or industry. However, further study should be conducted before its practical implementation.

Acknowledgements The authors thank Luleå Railway Research Centre (Järnvägstekniskt Centrum, Sweden) and Swedish Transport Administration (Trafikverket) for initiating the research study and providing financial support. This work was also partly supported by NSFC under a key project (Grand No. 71731008). We thank researchers involved in discussions of the concept, including from Luleå Tekniska Universitet (Sweden), Universidad Politecnica De Madrid (Spain), Technische Universiteit Delft (The netherlands), The University of Huddersfield (UK), Schneider Electric Espana SA (Spain), Infranord (Sweden), Integrasys SA (Spain), Digital Rail Limited (UK), and Limmat M\&M Sociedad Limitada (Spain). 


\section{References}

Asplund M, Lin J (2016). Evaluating the measurement capability of a wheel profile measurement system by using GR\&R. Measurement, 92: 19-27

Ben-Daya M, Kumar U, Murthy D N P (2015). Introduction to Maintenance Engineering: Modelling, Optimization and Management. New York: Wiley

Cai B, Liu Y H, Fan Q, Zhang Y W, Yu S L, Liu Z K, Dong X (2013). Performance evaluation of subsea BOP control systems using dynamic Bayesian networks with imperfect repair and preventive maintenance. Engineering Applications of Artificial Intelligence, 26(10): 2661-2672

Creamer L (2017). The Best Asset Management Services of 2017. Retrieved from http://uk.pcmag.com/cloud-services/79270/guide/ the-best-asset-management-services-of-2017, 2017-9-10

Galar D, Kumar U, Karim R (2017). Big data in railway operations and maintenance. Globe Railway Review, 4: 11-14

Figueres-Esteban M, Hughes P, Gulijk C (2016). Visual analytics for text-based railway incident reports. Journal of Safety Science, 89: 72-76

Karim R, Westerberg J, Galar D, Kumar U (2016). Maintenance analytics-The new know in maintenance. IFAC-PapersOnLine, 49(28): 214-219

Lee J, Kao H, Yang S (2014). Service innovation and smart analytics for Industry 4.0 and big data environment. Procedia CIRP, 16: 3-8

Lee J, Yang S, Lapira E, Kao H A, Yen N (2013). Methodology and framework of a cloud-based prognostics and health management system for manufacturing industry. Chemical Engineering Transactions, 33: 205-210

Lin J, Asplund M (2015). Bayesian semi-parametric analysis for locomotive wheel degradation using gamma frailties. Proceedings of the Institution of Mechanical Engineers, Part F: Journal of Rail and Rapid Transit, 229(3): 237-247

Lin J, Asplund M, Parida A (2014). Reliability analysis for degradation of locomotive wheels using parametric Bayesian approach. Quality and Reliability Engineering International, 30(5): 657-667

Lin J, Pulido J, Asplund M (2015). Reliability analysis for preventive maintenance based on classical and Bayesian semi-parametric degradation approaches using locomotive wheel-sets as a case study. Reliability Engineering \& System Safety, 134: 143-156

Meeker W, Hong Y (2014). Reliability meets Big data: Opportunities and challenges. Quality Engineering, 26: 102-116

Thaduri A, Galar D, Kumar U (2015). Railway assets: A potential domain for Big data analytics. Procedia Computer Science, 53: $457-$ 467

Yang S, Bagheri B, Kao H, Lee J (2015). A unified framework and platform for designing of cloud-based machine health monitoring and manufacturing systems. Journal of Manufacturing Science and Engineering, 137(4): 040914

Zhang L W, Lin J, Karim R (2015). An angle-based subspace anomaly detection approach to high-dimensional data: With an application to industrial fault detection. Reliability Engineering \& System Safety, 142: 482-497

Zhang L W, Lin J, Karim R (2017). Sliding window-based fault detection from high-dimensional data streams. IEEE Transactions on Systems, Man, and Cybernetics: Systems, 47(2): 289-303 